\title{
NONSTATIONARY FLOWS WITH VISCOUS HEATING EFFECTS
}

\author{
THIERRY CLOPEAU AND ANDRO MIKELIĆ
}

\begin{abstract}
The system of equations describing a nonstationary flow of a quasi-newtonian fluid, with temperature dependent viscosity and with the viscous heating, is considered. Existence of at least one weak solution is proved, i.e. we get existence of at least one velocity field having finite energy and existence of a nonnegative temperature field. Its regularity is a consequence of the $L^{1}$-forcing term generated by the viscous heating.
\end{abstract}

Key words: viscous heating, quasi-newtonian flow, weak solution.

Mathematics subject classification: AMS : 35Q35, 76 A05, 35J25, $76 \mathrm{E} 99$.

\section{INTRODUCTION}

In most polymer processing applications the temperature changes are important and cannot be neglected. An example is the manufacture of plastic objects involving processing operations as melting and cooling. It leads to considering the equations of a quasi-Newtonian flow with important viscous dissipation.

The starting point for our considerations is the following system of equations in a bounded domain $\Omega$ in $\mathbb{R}^{n}(n=2$ or 3$)$ with boundary $\Gamma \in C^{2}$ :

$$
\begin{gathered}
\operatorname{div} v=0 \quad \text { in } \quad \Omega \times] 0, I[ \\
\left.-\operatorname{div}\left\{\frac{1}{\eta^{0}} \nu(T,|D(v)|) D(v)\right\}+\nabla p=0 \quad \text { in } \quad \Omega \times\right] 0, I[ \\
\left.\mathbf{P} e ́\left\{\frac{\partial T}{\partial t}+v \nabla T\right\}-\Delta T=\frac{\mathbf{G} n}{2 \eta^{0}} \nu(T,|D(v)|)|D(v)|^{2} \quad \text { in } \quad \Omega \times\right] 0, I[,
\end{gathered}
$$

where we denote the rate of strain tensor $D(\tilde{v}) \equiv \operatorname{sym} \nabla \tilde{v}$. Its second invariant is denoted by $|D(\tilde{v})|^{2}, \eta^{0}$ is the characteristic viscosity, $\mathbf{P} e ́$ is the Péclet number and $\mathbf{G} n$ is the heat generation number. The unknowns are $T$ the temperature, $v$ the velocity and $p$ the pressure.

For a detailed discussion on the rheological aspects of the problem we refer to Bird, Armstrong, Hassager [2]. A derivation using the basic conservation laws of continuum mechanics can be found in Baranger, Mikelić [1].

System (1)-(3) is subject to the following boundary and initial conditions:

$$
\begin{array}{ccccc}
v=v_{w} & \text { on } & \Gamma \times] 0, I[ \\
T(x, 0)=T_{0} \quad \text { in } \quad \Omega ; & & \left.T=T_{w} \quad \text { on } \quad \Gamma \times\right] 0, I[
\end{array}
$$

The stationary variant of problem (1)-(5) is considered in Baranger, Mikelić [1] and for a large class of viscosity laws $\nu(T,|D(v)|)$, satisfying ellipticity and continuity conditions, existence of at least one weak solution was proved. 
In this note we extend those results from the stationary to the nonstationary case. In order to simplify the technicalities we can take, for example, $\nu$ as product of the Arrhenius law for the temperature and a power law for $|D(v)|$, i.e.

$$
\nu(z, \xi)=\mu_{0} \exp \left[\frac{E}{R}\left(\frac{1}{z}-\frac{1}{T_{1}}\right)\right] \xi^{r-2}, \quad \forall z>0 \quad \text { and } \quad \forall \xi>0,
$$

where $\mu_{0}, E, R$ and $T_{1}$ are some positive constants and $1<r$ is a real number. We will see that this viscosity verifies sufficient conditions for the existence of at least one solution for the problem.

\section{Statement of the Results}

Now we are in situation to state precisely our problem. Let us specify the assumptions on the boundary conditions. We suppose

$$
\left.v_{w} \in L^{\infty}\left(0, I ; W^{1-\frac{1}{r}, r}(\Gamma)^{n}\right), \quad \text { with } \quad \int_{\Gamma} v_{w} \cdot \vec{n} d \sigma=0 \quad \text { (a.e.) on } \quad\right] 0, I[\text { ( }
$$

and

$$
\begin{gathered}
\left.T_{w} \in L^{\infty}(\Gamma \times] 0, I[) \cap H^{1}(\Gamma \times] 0, I[), T_{w} \geq C_{T}>0 \text { (a.e.) on } \Gamma \times\right] 0, I[, \\
T_{0} \in L^{\infty}(\Omega), \quad T_{0} \geq C_{T}>0 \quad \text { (a.e.) on } \Omega .
\end{gathered}
$$

Then there exists $\tilde{v}_{w} \in L^{\infty}\left(0, I ; W^{1, r}(\Omega)^{n}\right)$ a divergence free lift of the injection velocity $v_{w}$, and $\tilde{T}_{w} \in L^{q}\left(0, I ; W^{1, q}(\Omega)\right)$ a lift of the boundary temperature $T_{w}$.

The functional space $V_{r}$ is given by

$$
V_{r}=\left\{z \in W_{0}^{1, r}(\Omega)^{n}: \operatorname{div} z=0 \text { in } \Omega\right\},
$$

where $r$ in (7) and (10) is connected with the appropriate law for the viscosity.

In analogy with the stationary case we introduce

Definition 1 . We say that $\{v, T\}$ is a weak solution of (1)-(5) if

$$
\begin{gathered}
v-\tilde{v}_{w} \in L^{\gamma}\left(0, I ; V_{r}\right), \quad \forall \gamma<+\infty \\
\left.\int_{\Omega} \nu\left(T,\left|D\left(v+\tilde{v}_{w}\right)\right|\right) D\left(v+\tilde{v}_{w}\right): D(\varphi)=0, \forall \varphi \in V_{r}, \text { (a.e) on }\right] 0, I[(11) \\
\left.T-\tilde{T}_{w} \in L^{q}\left(0, I ; W_{0}^{1, q}(\Omega)\right), \quad \forall q \in\right] 1,(n+2) /(n+1)[ \\
\left.T \geq C_{T} \quad \text { a.e. in } \Omega \times\right] 0, I[
\end{gathered}
$$

and

$$
\begin{gathered}
-\int_{0}^{I} \int_{\Omega} \mathbf{P} \dot{e}\left\{T \frac{\partial \varphi}{\partial t}+\left(v+\tilde{v}_{w}\right) T \nabla \varphi\right\}+\int_{0}^{I} \int_{\Omega} \nabla T \nabla \varphi-\int_{\Omega} \mathbf{P} \dot{e} T_{0} \varphi(x, 0) \\
=\frac{\mathbf{G} n}{2 \eta^{0}} \int_{0}^{I} \int_{\Omega} \nu\left(T,\left|D\left(v+\tilde{v}_{w}\right)\right|\right)\left|D\left(v+\tilde{v}_{w}\right)\right|^{2} \varphi \\
\left.\forall \varphi \in C^{\infty}(\Omega \times[0, I]), \quad \varphi=0 \quad \text { on } \Omega \times\{I\} \cup \Gamma \times\right] 0, I[
\end{gathered}
$$

We can now state the main result of this paper. 
THEOREM 2. Let us assume (6)-(9). Then there exists a weak solution $\{v, T\}$ to problem (11)-(13).

\section{Auxiliary Results}

We start with some property of the viscosity of the fluid.

Lemma 3. Let $\nu$ be given by (6). Then $\nu \in C\left(\mathbb{R} \times \mathbb{R}_{s}^{n^{2}}\right)$ and there exist constants $\bar{c}_{\nu}(r), C_{\nu}(r)$ and $\bar{C}_{\nu}(r)$ such that

$$
\begin{gathered}
\left|\nu\left(w_{1},|A|\right)-\nu\left(w_{2},|A|\right)\right| \leq \bar{c}_{\nu}(r) \Theta\left(\left|w_{1}-w_{2}\right|\right)|A|^{r-2} \\
\forall w_{1}, w_{2} \in \mathbb{R}^{+}, \quad \forall A \in \mathbb{R}_{s}^{n^{2}}, \quad \text { with } \quad \Theta \in C_{b}(\mathbb{R}), \quad \Theta(0)=0 \\
\left(\begin{array}{ll}
\nu\left(z,\left|A_{1}\right|\right) A_{1}-\left(\nu\left(z,\left|A_{2}\right|\right) A_{2}\right):\left(A_{1}-A_{2}\right) \geq \\
C_{\nu}(r) \frac{\left|A_{1}-A_{2}\right|^{2}}{\left\{\left|A_{1}\right|+\left|A_{2}\right|\right\}^{r-2}} \quad \text { for } \quad 1<r<2 \\
\forall z \in \mathbb{R}^{+}, \quad \forall A_{1}, A_{2} \in \mathbb{I R}_{s}^{n^{2}}
\end{array}\right.
\end{gathered}
$$

and

$$
\begin{gathered}
\frac{1}{|B|}\left|\left(\nu\left(z,\left|A_{1}\right|\right) A_{1}-\nu\left(z,\left|A_{2}\right|\right) A_{2}\right): B\right| \leq \\
\left\{\begin{array}{l}
\bar{C}_{\nu}(r){\frac{\left|A_{1}-A_{2}\right|}{\left\{\left|A_{1}\right|+\left|A_{2}\right|\right\}}}^{2-r} \quad \text { for } r \geq 2 \\
\bar{C}_{\nu}(r)\left|A_{1}-A_{2}\right|^{r-1} \quad \text { for } 1<r<2
\end{array}\right. \\
\forall z \in \mathbb{R}^{+}, \quad \forall A_{1}, A_{2} \text { and } B \in \mathbb{R}_{s}^{n^{2}} .
\end{gathered}
$$

Proof. See e.g. [5].

The proof requires regularization of the velocity field. We are going to use the following classical result:

Lemma 4. Let $\omega$ be a mollifier in $\mathbb{R}^{n}$ and let $u \in L_{d i v}^{\alpha}(\Omega)^{n}=\{u \in$ $L^{\alpha}(\Omega)^{n}$, div $u \in L^{\alpha}(\Omega), u \cdot \nu=0$ on $\left.\Gamma\right\}$. Furthermore, let $u_{h}$ (the regularization of $u$ ) be defined by

$$
u_{h}(x)=h^{-n} \int_{\Omega} \omega\left(\frac{x-y}{h}\right) u(y) d y \quad, \quad 0<h<\operatorname{dist}(x, \partial \Omega) .
$$

Let us extend $u$ to $\mathbb{I}^{n}$ by zero, then $u_{h} \longrightarrow u$ in $L_{d i v}^{\alpha}(\Omega)^{n}$ as $h \longrightarrow 0$.

Finally, let $z \in W_{0}^{1, q}(D),+\infty>q>1$, with $D \supset \supset$. Then $z_{h} \longrightarrow z$ in $W^{1, q}(\Omega)$ as $h \longrightarrow 0$. 
Proof. See e.g. [4], p. 144-151.

In order to prove the theorem we regularize the boundary conditions for velocity and for temperature and the convection term in the equation for $T$. Consequently, we consider the folowing regularized problem

Definition 5. We say that $\left\{v^{h}, T^{h}\right\}$ is a weak solution of the regularized problem if

$$
\begin{aligned}
& v^{h}-\tilde{v}_{w h} \in L^{\infty}\left(0, I ; V_{r}\right), \\
& \int_{\Omega} \nu\left(T^{h},\left|D\left(v^{h}+\tilde{v}_{w h}\right)\right|\right) D\left(v^{h}+\tilde{v}_{w h}\right): D(\varphi)=0 \\
& \left.\forall \varphi \in V_{r} \text { (a.e.) on }\right] 0, I[\text {, } \\
& T^{h}-\tilde{T}_{w h} \in \bigcap_{1 \leq q<\frac{n}{n-1}} \bigcap_{1 \leq b<\frac{2 q}{(n+1) q-n}} L^{b}\left(0, I ; W_{0}^{1, q}(\Omega)\right), \\
& \left.T^{h} \geq C_{T} \text { a.e. in } \Omega \times\right] 0, I[\text {, } \\
& -\int_{0}^{I} \int_{\Omega} \mathbf{P} \dot{e}\left\{T^{h} \frac{\partial \varphi}{\partial t}+\left(v_{h}^{h}+\tilde{v}_{w h}\right) T^{h} \nabla \varphi\right\} \\
& +\int_{0}^{I} \int_{\Omega} \nabla T^{h} \nabla \varphi+\int_{\Omega} \mathbf{P} e ́ T_{0 h} \varphi(x, 0) \\
& =\frac{\mathbf{G} n}{2 \eta^{0}} \int_{0}^{I} \int_{\Omega} \nu\left(T^{h},\left|D\left(v^{h}+\tilde{v}_{w h}\right)\right|\right)\left|D\left(v^{h}+\tilde{v}_{w h}\right)\right|^{2} \varphi, \\
& \left.\forall \varphi \in C^{\infty}(\Omega \times[0, I]), \quad \varphi=0 \quad \text { on } \Omega \times\{I\} \cup \Gamma \times\right] 0, I[,
\end{aligned}
$$

where $u_{h}$ is the regularization with the respect to the space variables of a given function $u$.

We have

Proposition 6. Let us assume (6)-(9). Then there exists a weak solution $\left\{v^{h}, T^{h}\right\}$ to the problem (18)-(20), $\left.\forall h \in\right] 0, h_{0}[$.

Proof. We apply Schauder's fixed point theorem in a closed convex set $C_{R}=$ $B_{R} \cap\left\{z \geq C_{T} \quad(\right.$ a.e. $\left.)\right\} \subset L^{\beta}\left(0, I ; W^{1, \beta}(\Omega)\right)$, where $B_{R}$ is a closed ball and $\beta \in] 1, \frac{n+2}{n+1}\left[\right.$, to the mapping $\Upsilon: z \longrightarrow T_{z}$, constructed as follows :

Let $z \in L^{\beta}\left(0, I ; W^{1, \beta}(\Omega)\right), z \geq C_{T}$ and denote by $v_{z}$ the unique solution to the problem:

Find $v_{z} \in L^{\infty}\left(0, I ; L^{r}(\Omega)^{n^{2}}\right)$ such that

$$
\int_{\Omega} \nu\left(z,\left|D\left(v_{z}+\tilde{v}_{w h}\right)\right|\right) D\left(v_{z}+\tilde{v}_{w h}\right): D(\varphi)=0, \quad \forall \varphi \in V_{r} .
$$

After inserting $\varphi=v_{z}$ as test function in (21) we get

$$
\left\|D\left(v_{z}\right)\right\|_{L^{\infty}\left(0, I ; L^{r}(\Omega)^{n^{2}}\right)} \leq C,
$$

with $C=C(\Omega, I, n, r)$. 
In addition, we easily get the existence of the corresponding pressure field $p_{z} \in L^{\infty}\left(0, I ; L^{r^{\prime}}(\Omega) / I R\right), \sigma_{z}=\mu\left(z,\left|D\left(v_{z}+\tilde{v}_{w h}\right)\right|\right) D\left(v_{z}+\tilde{v}_{w h}\right)-p_{z} I \in$ $L^{\infty}\left(0, I ; L^{r^{\prime}}(\Omega)^{n^{2}}\right)$, and $\sigma_{z}: D\left(v_{w}+\tilde{v}_{w h}\right) \in L^{\infty}\left(0, I ; L^{1}(\Omega)\right)$, with bounds independent of $z$ and $h$.

Now we consider the problem

$$
\left\{\begin{array}{l}
\mathbf{P} \dot{e}\left\{\frac{\partial T_{z}}{\partial t}+\left(v_{z h}+\tilde{v}_{w h}\right) \nabla T_{z}\right\}-\Delta T_{z}= \\
\left.=\frac{\mathbf{G} n}{2 \eta^{0}} \nu\left(z,\left|D\left(v_{z}+\tilde{v}_{w h}\right)\right|\right)\left|D\left(v_{z}+\tilde{v}_{w h}\right)\right|^{2} \quad \text { in } \quad \Omega \times\right] 0, I[ \\
\left.T_{z}(x, 0)=T_{0 h} \quad \text { in } \Omega, \quad T_{z}=T_{w h} \quad \text { on } \quad \Gamma \times\right] 0, I[
\end{array}\right.
$$

Since the right hand side is in $L^{1}(\Omega)$ we are not able to use the variational method. One possibility is to use directly the results from Boccardo, Gallouet [3]. However, problem (23) is linear and we are able to use the linear theory. Our idea is to use the particular structure of the problem (23) and to decompose it into two simpler problems. Then the elementary theory gives the optimal results.

We start with an auxiliary problem:

$$
\left\{\begin{array}{l}
\mathbf{P} \dot{e}\left\{\frac{\partial T_{r}^{(1)}}{\partial t}+\left(v_{z h}+\tilde{v}_{w h}\right) \nabla T_{r}^{(1)}\right\}-\Delta T_{r}^{(1)}= \\
\left.=\frac{\mathbf{G} n}{2 \eta^{0}} \nu\left(z,\left|D\left(v_{z}+\tilde{v}_{w h}\right)\right|\right)\left|D\left(v_{z}+\tilde{v}_{w h}\right)\right|^{2} \text { in } \quad \Omega \times\right] 0, I[ \\
\left.T_{r}^{(1)}(x, 0)=0 \quad \text { in } \Omega \times\right] 0, I\left[, \quad T_{r}^{(1)}=0 \quad \text { on } \quad \Gamma \times\right] 0, I[
\end{array}\right.
$$

The left hand side of (24) defines Green's operator :

$$
f \in L^{2}\left(0, I ; H^{-1}(\Omega)\right) \longrightarrow G f \in L^{2}\left(0, I ; H_{0}^{1}(\Omega)\right) .
$$

When $v_{z h}+\tilde{v}_{w h} \in L^{\infty}(] 0, I\left[; L^{a}(\Omega)^{n}\right), a>n$, the classical theorem of Nash and Moser (see e.g. Ladyzhenskaya, Solonnikov, Ural'ceva [6]) implies that the adjoint operator to Green's operator is compact from $L^{b / 2}\left(0, I ; L^{q / 2}(\Omega)\right)$ to $C(\bar{\Omega} \times[0, I])$ and from $L^{b}\left(0, I ; W^{-1, q}(\Omega)\right)$ to $C(\bar{\Omega} \times$ $[0, I])$, for $q>n$ and $b>2 q /(q-n)$. Then Green's operator is compact from $M_{b}(] 0, I[\times \Omega)=\left(C_{0}(] 0, I[\times \Omega)\right)^{\prime}$ to $L^{b^{\prime}}\left(0, I ; W_{0}^{1, q^{\prime}}(\Omega)\right)$ equipped with the topology $\sigma_{b}\left(M_{b}(] 0, I[\times \Omega), C_{0}(] 0, I[\times \Omega)\right)$. Therefore, using the standard duality argument we obtain that Green's operator is compact from $L^{1}(\Omega \times] 0, I[)$ to $L^{b^{\prime}}\left(0, I ; W_{0}^{1, q^{\prime}}(\Omega)\right)$, for $q^{\prime}<n /(n-1)$ and $b^{\prime}<2 q^{\prime} /\left((n+1) q^{\prime}-n\right)$.

In the special case $b^{\prime}=q^{\prime}$ Green's operator is compact from $L^{1}(\Omega \times] 0, I[)$ to $L^{l}\left(0, I ; W_{0}^{1, l}(\Omega)\right)$, for $l<(n+2) /(n+1)$, which corresponds to the result in Boccardo, Gallouët [3].

Therefore, there exists a unique solution

$$
T_{r}^{(1)} \in \bigcap_{1 \leq q<\frac{n}{n-1}} \bigcap_{1 \leq b<\frac{2 q}{(n+1) q-n}} L^{b}\left(0, I ; W_{0}^{1, q}(\Omega)\right)
$$

for (24) and it is bounded in the corresponding norm by a constant $C$ depending on $\Omega, I, n$, and $h$. Because of (22), this constant depends on $h$ only in the case $n=3$ and $1<r \leq 3 / 2$. Otherwise, $\left\|v_{z h}\right\|_{L^{\infty}\left(10, I\left[; L^{a}(\Omega)^{n}\right)\right.}, a>n$, is uniformly bounded independently of $h$. Positivity of the right hand side implies $T_{r}^{(1)} \geq 0$ (a.e.). 
Finally, the linear parabolic problem

$$
\left\{\begin{array}{ccc}
\mathbf{P} e ́\left\{\frac{\partial T_{r}^{(2)}}{\partial t}+\left(v_{z h}+\tilde{v}_{w h}\right) \nabla T_{r}^{(2)}\right\}-\Delta T_{r}^{(2)}=0 & \text { in } \quad \Omega \times] 0, T[ \\
\left.T_{r}^{(2)}(x, 0)=T_{0 h} \quad \text { in } \Omega ; \quad T_{r}^{(2)}=T_{w h} \quad \text { on } \quad \Gamma \times\right] 0, T[
\end{array}\right.
$$

has a unique solution $T_{r}^{(2)} \in W(0, I)$. Now $T_{z}=T_{r}^{(1)}+T_{r}^{(2)}$ is a unique solution for (23). The classical argument gives $T_{z} \geq C_{T}$ (a.e.) and

$\left\|T_{z}\right\|_{L^{\beta}\left(0, I ; W^{1, \beta}(\Omega)\right)}$ is uniformly bounded by a constant which does not depend on $z$ or $v_{z}$, but can depend on $h$.

Now we easily find $R>0$ such that $\Upsilon\left(C_{R}\right) \subset C_{R} \subset L^{\beta}\left(0, I ; W^{1, \beta}(\Omega)\right)$, $\beta \in] 1, \frac{n+2}{n+1}\left[\right.$ and $C_{R}=B_{R} \cap\left\{z \geq C_{T} \quad\right.$ (a.e.) $\}$. Furthermore, we need only to show that $\Upsilon$ is continuous, since compactness of Green's operator implies its compactness.

Let $\left\{z_{j}\right\}$ be a sequence in $\left.L^{\beta}\left(0, I ; W^{1, \beta}(\Omega)\right), \beta \in\right] 1, \frac{n+2}{n+1}[$ converging towards $z$. Then the corresponding functions $\left\{v_{j}\right\}$ defined by (21) are uniformly bounded and we are able to extract a subsequence $\left\{v_{k}\right\}$ converging weakly in $L^{\gamma}\left(0, I ; V_{r}\right), \gamma<+\infty$, towards $v_{z}$, being a unique solution for (21) corresponding to $z$. The uniqueness implies in fact the convergence of whole sequence.

At this stage we subtract equations for $v_{j}$ and $v_{z}$ and get

$$
\begin{gathered}
\int_{\Omega}\left\{\nu\left(z_{j},\left|D\left(v_{j}+\tilde{v}_{w h}\right)\right|\right) D\left(v_{j}+\tilde{v}_{w h}\right)\right. \\
\left.-\nu\left(z_{j},\left|D\left(v_{z}+\tilde{v}_{w h}\right)\right|\right) D\left(v_{z}+\tilde{v}_{w h}\right)\right\}: D(\varphi) \\
=\int_{\Omega}\left\{\nu\left(z,\left|D\left(v_{z}+\tilde{v}_{w h}\right)\right|\right)-\nu\left(z_{j},\left|D\left(v_{z}+\tilde{v}_{w h}\right)\right|\right)\right\} D\left(v_{z}+\tilde{v}_{w h}\right): D(\varphi), \\
\forall \varphi \in V_{r}
\end{gathered}
$$

We choose $\varphi=v_{j}-v_{z} \in V_{r}$ as a test function.

Then we obtain

$$
\begin{gathered}
\int_{0}^{I} \mid \int_{\Omega}\left\{\nu\left(z,\left|D\left(v_{z}+\tilde{v}_{w h}\right)\right|\right)-\nu\left(z_{j},\left|D\left(v_{z}+\tilde{v}_{w h}\right)\right|\right)\right\} \\
D\left(v_{z}+\tilde{v}_{w h}\right):\left.D\left(v_{j}-v_{z}\right)\right|^{\gamma} \\
\leq C|| D\left(v_{j}-v_{z}\right) \|_{L^{\infty}\left(0, I ; L^{r}(\Omega)^{n^{2}}\right)} \int_{0}^{I}\left(\int_{\Omega} \Theta\left(\left|z_{j}-z\right|\right)^{r^{\prime}}\left|D\left(\tilde{v}_{w h}\right)\right|^{r}\right)^{\frac{\gamma}{r^{\prime}}}
\end{gathered}
$$

Hence by Lebesgue's dominated convergence theorem the expression (27) tends to zero.Finally,

$$
\begin{gathered}
\int_{0}^{I} \mid \int_{\Omega}\left\{\nu\left(z_{j},\left|D\left(v_{j}+\tilde{v}_{w h}\right)\right|\right) D\left(v_{j}+\tilde{v}_{w h}\right)-\nu\left(z_{j},\left|D\left(v_{z}+\tilde{v}_{w h}\right)\right|\right) D\left(v_{z}+\tilde{v}_{w h}\right)\right\} \\
:\left.D\left(v_{j}-\tilde{v}_{w h}\right)\right|^{\gamma} \rightarrow 0
\end{gathered}
$$

and $v_{j} \longrightarrow v_{z}$ in $L^{\gamma}\left(0, I ; V_{r}\right), \gamma<+\infty$.

After establishing the strong convergence of $D\left(v_{j}\right)$ passing to the limit in (23) is obvious. By uniqueness the whole sequence $\left\{T_{z_{j}}\right\}$ converges and sequential continuity of $\Upsilon$ is proved. 
Now we are going to pass to the limit as $h \longrightarrow 0$. Firstly, we need an a priori estimate for $T^{h}$. As already remarked in Lewandowski [7] the transport term $\left(v_{h}^{h}+\tilde{v}_{w h}\right) \nabla T^{h}$ does not give any contribution in estimating $T^{h}$ by truncation. The estimate follows using the calculations from Boccardo, Gallouët [3]. For the confort of the reader we give some details. We have

Proposition 7. Let $T^{h}$ be defined in proposition 1, then

$$
\left.\left\|T^{h}\right\|_{L^{q}\left(0, I ; W^{1, q}(\Omega)\right)} \leq C, \quad \forall q \in\right] 1,(n+2) /(n+1)[.
$$

Proof. Without loosing the generality, we take:

$\mathbf{P e ́}=1, f^{h}=\frac{\mathbf{G} n}{2 \eta^{0}} \nu\left(T^{h},\left|D\left(v^{h}+\tilde{v}_{w h}\right)\right|\right)\left|D\left(v^{h}+\tilde{v}_{w h}\right)\right|^{2}$ and $u=v_{h}^{h}+\tilde{v}_{w h}$.

We search $T^{h}$ in the form $T^{h}=T_{(1)}^{h}+T_{(2)}^{h}$ with $T_{(1)}^{h}$ a solving

$$
\left\{\begin{array}{ccc}
\frac{\partial T_{(1)}^{h}}{\partial t}+u \nabla T_{(1)}^{h}-\Delta T_{(1)}^{h}=f^{h} & \text { in } \quad \Omega \times] 0, T[ \\
T_{(1)}^{h}(x, 0)=0 \quad \text { in } \Omega ; \quad T_{(1)}^{h}=0 & \text { on } \quad \Gamma \times] 0, T[
\end{array}\right.
$$

and $T_{(2)}^{h}$ a solving

$$
\left\{\begin{array}{cc}
\frac{\partial T_{(2)}^{h}}{\partial t}+u \nabla T_{(2)}^{h}-\Delta T_{(2)}^{h}=0 & \text { in } \quad \Omega \times] 0, T[ \\
T_{(2)}^{h}(x, 0)=T_{0 h} \quad \text { in } \Omega ; \quad T_{(2)}^{h}=T_{w h} & \text { on } \quad \Gamma \times] 0, T[
\end{array}\right.
$$

Obviously there exists a constant $C$ such that $\left\|T_{(2)}^{h}\right\|_{L^{q}\left(0, I ; W^{1, q}(\Omega)\right)} \leq C$ for $h$ sufficiently small.

Let $\left\{f_{k}^{h}\right\} \in C_{0}^{\infty}(\Omega \times] 0, I[)$ be an approximating sequence for $f^{h}$ in $L^{\infty}\left(0, I ; L^{1}(\Omega)\right)$. It should be noticed that $f^{h} \geq 0$ and we can take $f_{k}^{h} \geq 0$ as well. Let $T_{k}^{h}$ be the regularized solution given by (29) with $f_{k}^{h}$ as the right hand side.Then $T_{k}^{h}$ satisfies the following weak formulation :

$$
\begin{gathered}
\int_{0}^{I} \int_{\Omega}\left\{\frac{\partial T_{k}^{h}}{\partial t} \varphi+u \nabla T_{k}^{h} \varphi\right\}+\int_{0}^{I} \int_{\Omega} \nabla T_{n}^{h} \nabla \varphi=\int_{0}^{I} \int_{\Omega} f_{n}^{h} \varphi \\
\forall \varphi \in L^{2}\left(0, I ; H_{0}^{1}(\Omega)\right)
\end{gathered}
$$

Let $\psi_{m}$ be the real function defined on $\mathbb{R}^{+}$by

$$
\psi_{m}(s)=\left\{\begin{array}{clc}
0 & \text { if } & s \leq m \\
s-m & \text { if } & m<s \leq m+1 \\
1 & \text { if } & s>m
\end{array}\right.
$$

and let $\phi_{m}(s)=\int_{0}^{s} \psi_{m}(r) d r$

Let us take $\psi_{0}\left(T_{k}^{h}\right) \chi_{(0, t)}$ as a test function in (31) and integrate by parts. Then after using that $f_{k}^{h}$ is bounded independently of $h$ and $k$ in $L^{\infty}\left(0, I ; L^{1}(\Omega)\right)$, and noting that

$$
\int_{0}^{t} \int_{\Omega} u \nabla T_{k}^{h} \psi_{0}\left(T_{k}^{h}\right)=\int_{0}^{t} \int_{\Omega} u \nabla \phi_{0}\left(T_{k}^{h}\right)=-\int_{0}^{t} \int_{\Omega} \phi_{0}\left(T_{k}^{h}\right) \operatorname{div} u=0,
$$

we obtain

$$
\left\|T_{k}^{h}\right\|_{L^{\infty}\left(0, I ; L^{1}(\Omega)\right)} \leq C
$$

The choice of $\psi_{m}\left(T_{k}^{h}\right)$ as a test fonction yields 


$$
\int_{B_{m}}\left|\nabla T_{k}^{h}\right|^{2} \leq C
$$

with $B_{m}=\{(x, t) \in \Omega \times] 0, I\left[; m \leq T_{k}^{h}(x, t) \leq m+1\right\}$ and $C$ independent of $m, k$ and $h$. Let $r=\frac{n+1}{n} q$, then we have

$$
\int_{B_{m}}\left|\nabla T_{k}^{h}\right|^{q} \leq C\left(\text { meas } B_{m}\right)^{1-q / 2} \leq C\left(\int_{B_{m}}\left|T_{k}^{h}\right|^{r}\right)^{1-q / 2} \frac{1}{m^{r(1-q / 2)}}
$$

which gives

$$
\begin{aligned}
\int_{0}^{I} \int_{\Omega}\left|\nabla T_{k}^{h}\right|^{q} & \leq C\left(c\left(m_{0}\right)+\sum_{m=m_{0}}^{\infty}\left(\int_{B_{m}}\left|T_{k}^{h}\right|^{r}\right)^{1-q / 2} \frac{1}{m^{r(1-q / 2)}}\right) \\
& \leq C\left(c\left(m_{0}\right)+\left(\int_{0}^{I} \int_{\Omega}\left|T_{k}^{h}\right|^{r}\right)^{1-q / 2}\left(\sum_{m=m_{0}}^{\infty} \frac{1}{m^{r(1-q / 2)}}\right)^{q / 2}\right)
\end{aligned}
$$

Hölder's inequality and (34) give

$$
\left\|T_{k}^{h}\right\|_{L^{r}\left(0, I ; L^{r}(\Omega)\right)}^{r} \leq C\left\|T_{k}^{h}\right\|_{L^{q}\left(0, I ; L^{q^{*}}(\Omega)\right)}^{q}
$$

with $q^{*}=q /(1-r+q)$. Now Sobolev imbedding theorem implies

$$
\left\|T_{k}^{h}\right\|_{L^{q}\left(0, I ; L^{q^{*}}(\Omega)\right)}^{q} \leq C \int_{0}^{I} \int_{\Omega}\left|\nabla T_{k}^{h}\right|^{q}
$$

and consequently

$$
\left\|T_{k}^{h}\right\|_{L^{q}\left(0, I ; L^{q^{*}}(\Omega)\right)}^{q} \leq C\left(c\left(m_{0}\right)+\left\|T_{k}^{h}\right\|_{L^{q}\left(0, I ; L^{q^{*}}(\Omega)\right)}^{q(1-q / 2)}\left(\sum_{m=m_{0}}^{\infty} \frac{1}{m^{r(1-q / 2)}}\right)^{q / 2}\right)
$$

A proper choice of $m_{0}$ gives the estimate

$$
\left\|T_{k}^{h}\right\|_{L^{q}\left(0, I ; L^{q^{*}}(\Omega)\right)} \leq C
$$

Finally (3),(37) and (40) give the result.

\section{Proof of the theorem}

Let us introduce a number $\alpha$ by

$$
\alpha= \begin{cases}\min \{(n+2) /(n+1), n q r /(n(q+r)-2 r q)\} & \text { for } \quad 1<r<n \\ (n+2) /(n+1) & \text { for } \quad r \geq n\end{cases}
$$

Then using (28) and the equation (23) we deduce the following estimate:

$$
\left.\left\|\frac{d T^{h}}{d t}\right\|_{L^{q}\left(0, I ; W^{-1, \alpha}(\Omega)\right)+L^{1}(\Omega \times] 0, I[)} \leq C, \quad \forall q \in\right] 1,(n+2) /(n+1)[.
$$

Now $\left\{T^{h}\right\}$ is relatively compact in $L^{1}(\Omega \times] 0, I[)$ by a compactness lemma of Aubin's type (see e.g. Simon [8]). After extracting a subsequence, denoted by the same symbol, we get

$$
\begin{array}{cccl}
v^{h} \rightarrow v & \text { weakly } & \text { in } & L^{\gamma}\left(0, I ; V_{r}\right), \gamma<+\infty \\
T^{h} \longrightarrow T & \text { strongly } & \text { in } & L^{q}\left(0, I ; W^{1, q}(\Omega)\right), 1 \leq q<\frac{n+2}{n+1}
\end{array}
$$


Using Minty's lemma we write equation (23) as

$$
\int_{\Omega} \mu\left(T^{h},\left|D\left(\varphi+\tilde{v}_{w h}\right)\right|\right) D\left(\varphi+\tilde{v}_{w h}\right): D\left(\varphi+\tilde{v}_{w h}-v^{h}\right) \geq 0 \quad \forall \varphi \in V_{r}
$$

Then passing to the limit is obvious and we get

$$
\int_{0}^{I} \int_{\Omega} \mu(T,|D(v)|) D(v): D(\varphi) \zeta=0 \quad \forall \varphi \in V_{r}, \quad \zeta \in C^{\infty}(0, I) .
$$

At this stage we proceed as in the proof of continuity of the operator $\Upsilon$ and, after passing once more to the subsequence, obtain

$$
v^{h} \longrightarrow v \quad \text { strongly in } L^{\gamma}\left(0, I ; V_{r}\right), \quad \forall \gamma<+\infty
$$

Now passing to the limit in (20) is obvious and we conclude that $\{v, T\}$ is a solution for (11)-(13).

\section{REFERENCES}

[1] J.BARANger, A.Mikelić , Stationary Solutions to a Quasi-Newtonian Flow with Viscous Heating, Math. Models and Methods in Applied Sciences, 5 (1995), pp. 725738.

[2] R.B. Bird, R. C. Armstrong and O. Hassager, Dynamics of Polymeric Liquids, Vol.1, Fluid Mechanics, Wiley and Sons, New York,1987.

[3] L. Boccardo, Th. GallouËt, Non-linear Elliptic and Parabolic Equations Involving Measure Data, J. Funct. Anal., 87 (1989), pp. 149-169.

[4] D. Gilbarg, N. S. Trudinger , Elliptic Partial Differential Equations of Second Order, Springer, 1983.

[5] R. Glowinski, X. Marroco, Sur l'approximation par éléments finis d'ordre un et la résolution par pénalisation-dualité d'une classe des problèmes de Dirichlet nonlinéaires., R.A.I.R.O., R-2 (1975), pp. 47-76.

[6] O.A.Ladyzhenskaya, V.A.Solonnikov, N.N.Ural'Ceva, Linear and QuasiLinear Equations of Parabolic Type, American Math. Society, Providence, 1968.

[7] R. LEWANDOWSKI, The mathematical analysis of the coupling of a turbulent kinetic energy to the Navier-Stokes equation with an eddy viscosity, preprint.

[8] J.Simon, Compact sets in the space $L^{p}(0, T ; B)$, Ann. Mat. Pura Appl. , 196 (1987), pp. 65-96.

UMR CNRS 5585, Equipe D’Analyse Numérique Lyon - St-Etienne, BÂt. 101, Université Lyon 1, 43 BD. DU 11 NOVEMBRE 1918, 69622 VILLEURBANNE CEDEX, FRANCE 